\title{
Aspectos gerais sobre a obesidade infantil: uma revisão narrativa
}

\author{
General aspects of childhood obesity: a narrative review
}

Aspectos generales de la obesidad infantil: una revisión narrativa

Amanda Brandão Lopes ${ }^{1 *}$, Racquel Firpe Caetano1, Patrícia Pimenta Nunes ${ }^{1}$, Camila Godinho

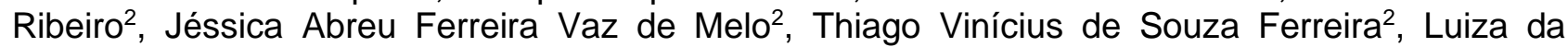
Nóbrega Tobias ${ }^{3}$, Bruna Pereira Silva ${ }^{4}$, Moisés Gustavo da Cunha ${ }^{5}$, Marianne Borges Freitas ${ }^{6}$.

\section{RESUMO}

Objetivo: Revisar e fornecer uma ampla abordagem sobre a obesidade infantil. Revisão bibliográfica: A obesidade na infância é considerada um problema grave de saúde pública mundial atualmente. O ganho de peso na faixa etária pediátrica está relacionado principalmente à ausência de aleitamento materno adequado, hábitos alimentares incorretos, com alimentação rica em açucares e gorduras e redução da prática de atividades físicas. A presença da obesidade predispõe a criança ao desenvolvimento de diversas doenças na vida adulta, como diabetes, dislipidemia e elevação da pressão arterial sistêmica. O diagnóstico é simples e deve ser realizado preferencialmente por um pediatra. A partir do momento em que for constato um aumento patológico do ganho de peso é necessário que o médico oriente os pais quanto aos riscos e a importância de uma alimentação balanceada e prática regular de exercícios físicos. Considerações finais: A obesidade deve ser tratada com objetivo de reduzir a morbimortalidade infantil e também na vida adulta. Portanto, a orientação profissional e promoção de políticas para a prevenção e tratamento dessa doença são de fundamentais importâncias no combate à obesidade infantil.

Palavras-chave: Obesidade infantil, Sobrepeso, Crianças.

\begin{abstract}
Objective: To review and provide a comprehensive approach to childhood obesity. Bibliography review: Childhood obesity is currently considered a serious public health problem worldwide. Weight gain in the pediatric age group is mainly related to the absence of adequate breastfeeding, incorrect eating habits, a diet rich in sugars and fats, and reduced physical activity. The presence of obesity predisposes children to the development of several diseases in adulthood, such as diabetes, dyslipidemia and elevated systemic blood pressure. Diagnosis is simple and should preferably be performed by a pediatrician. From the moment a pathological increase in weight gain is found, it is necessary for the physician to guide parents about the risks and the importance of a balanced diet and regular physical exercise. Final considerations: Obesity must be treated with the objective of reducing child and adult morbidity and mortality. Therefore, professional guidance and promotion of policies for the prevention and treatment of this disease are of fundamental importance in combating childhood obesity.
\end{abstract}

Key words: Childhood obesity, Overweight, Children.

\section{RESUMEN}

Objetivo: Revisar y brindar un abordaje integral de la obesidad infantil. Revisión bibliográfica: La obesidad infantil se considera actualmente un grave problema de salud pública en todo el mundo. El aumento de peso en el grupo de edad pediátrica se relaciona principalmente con la ausencia de una adecuada lactancia

\footnotetext{
${ }^{1}$ Faculdade de Minas de Belo Horizonte (FAMINAS-BH), Belo Horizonte - MG.

*E-mail: amandabrandaolopes@gmail.com

${ }^{2}$ Pontifícia Universidade Católica de Minas Gerais (PUC-MINAS), Betim - MG.

${ }^{3}$ Universidade José do Rosário Vellano (UNIFENAS-BH), Belo Horizonte - MG.

${ }^{4}$ Faculdade de Ciências Médicas e da Saúde de Juiz de For a (SUPREMA), Juiz de Fora - MG.

${ }^{5}$ Universidade Federal de Minas Gerais (UFMG), Belo Horizonte - MG.

${ }^{6}$ Universidade Federal de Uberlândia (UFU), Uberlândia - MG.
} 
materna, hábitos alimentarios incorrectos, una dieta rica en azúcares y grasas y la reducción de la actividad física. La presencia de obesidad predispone a los niños al desarrollo de varias enfermedades en la edad adulta, como diabetes, dislipidemia y presión arterial sistémica elevada. El diagnóstico es sencillo y preferiblemente debe ser realizado por un pediatra. Desde el momento en que se detecta un aumento patológico de la ganancia de peso, es necesario que el médico oriente a los padres sobre los riesgos y la importancia de una dieta equilibrada y ejercicio físico regular. Consideraciones finales: La obesidad debe tratarse con el objetivo de reducir la morbilidad y la mortalidad de niños y adultos. Por tanto, la orientación profesional y la promoción de políticas de prevención y tratamiento de esta enfermedad son de fundamental importancia en la lucha contra la obesidad infantil.

Palabras clave: Obesidade infantil, Sobrepeso, Crianças.

\section{INTRODUÇÃO}

A obesidade infantil é uma doença caracterizada por um aumento de gordura corporal. Ela é tratada atualmente como um dos maiores problemas de saúde pública em âmbito mundial com grande impacto na saúde das populações, uma vez que pode evoluir com doenças crônicas degenerativas do tipo não transmissíveis, como por exemplo Hipertensão Arterial Sistêmica (HAS), Diabetes Mellitus tipo 2 (DM2) e doenças cardiovasculares (PURNELL JQ, et al., 2018).

No Brasil, ela está presente em diferentes faixas econômicas, sendo mais comum no meio da classe social mais alta. Esta patologia é influenciada pela classe socioeconômica por meio da renda, educação e da ocupação e resulta em padrões comportamentais específicos que podem afetar a taxa metabólica, a ingestão calórica e gasto energético. À medida que alimentos saudáveis como carnes magras, peixes, frutas e vegetais, estão menos disponíveis para indivíduos com menor poder aquisitivo, maior o número de pessoas obesas (SOCIEDADE BRASILEIRA DE PEDIATRIA, 2019).

Segundo os dados da Organização Mundial da Saúde (OMS), nos últimos 10 anos a prevalência de obesidade infantil tem crescido em cerca de 10 a $40 \%$ na maioria dos países europeus. Segundo dados epidemiológicos, nas últimas décadas houve um aumento significativo da prevalência do excesso de peso e da obesidade em crianças e adolescentes em todo o mundo. As pesquisas do Instituto Brasileiro de Geografia e Estatística (IBGE) e do centroPesquisa de Orçamentos Familiares (POF) confirmam esse amento e descrevem a trajetória do aumento do peso das crianças brasileiras ao longo dos anos (CESARE MD, et al., 2019).

A obesidade pode então ser definida e classificada como excesso de gordura em todas as regiões do corpo, que pode ser causada por maus hábitos alimentares, doenças endócrino-metabólicas ou alterações genéticas. O sobrepeso pode surgir em qualquer período da vida do indivíduo, mas aparece principalmente no primeiro ano de vida, entre 5 e 6 anos de idade e na adolescência, no entanto a intervenção deve ser feita em qualquer período da vida, afim de evitar as complicações decorrentes do excesso de peso (PURNELL JQ, et al., 2018).

Como esta doença está cada mais prevalente em âmbito mundial, o objetivo desta revisão foi identificar, através de uma revisão da literatura, os aspectos gerais associados à obesidade na infância, destacando suas causas, fatores de risco, diagnóstico e tratamento abordando formas de manejo e prevenção.

\section{REVISÃO BIBLIOGRÁFICA}

\section{Etiologia e fisiopatologia da obesidade infantil}

A obesidade é de etiologia multifatorial com interação entre fatores metabólicos, genéticos, nutricionais, ambientais e psicossociais. Além disso, as mudanças no estilo de vida parecem estar envolvidas na sua gênese, resultando em um balanço energético positivo. As mudanças nos hábitos alimentares e de consumo ao decorrer do tempo, refletem as modificações sociais, econômicas e demográficas em uma determinada população. A urbanização e o avanço das tecnologias instituíram diversos novos padrões de comportamento nas sociedades, o que vem desempenhando papel importante (PURNELL JQ, et al., 2018). 
As características do indivíduo com excesso de peso são resultadas da interação entre inúmeros genes, determinando uma herança genética de caráter poligênico na obesidade. Um estudo realizado entre gêmeos ou crianças adotadas e seus pais mostram clara influência genética no índice de massa corporal, na suscetibilidade ao ganho de peso, no gasto energético, na distribuição anatômica da gordura (WANG Y, et al., 2017).

O crescente aumento no número de pessoas obesas no mundo pode indicar uma importante participação do ambiente no programa genético. Mudanças ocorridas nos hábitos alimentares e no estilo de vida, somadas ao aumento do sedentarismo e consumo elevado de alimentos com alta densidade energética, explicam esse fato. Atualmente já existem mais de 400 genes isolados capazes de codificar componentes que participam da regulação do peso corporal. Dentre esses componentes, existem os que agem preferencialmente na ingestão alimentar, no gasto energético e os que modulam essas ações ou atuam nos dois mecanismos (STANFORD FC, et al., 2017).

\section{Fatores de risco associados à obesidade infantil}

A obesidade resulta de um desequilíbrio entre gasto energético reduzido e ganho energético elevado, além de diversos fatores de interação. Na dieta, o ganho energético está relacionado às escolhas alimentares incorretas e ao ambiente familiar obesogênico. O gasto energético, por sua vez, está associado à prática de atividade física ou ao sedentarismo, ao tempo de tela e ao sono. Dentre os diversos fatores de interação temse o tipo epigenéticos, genéticos, a adiposidade materna, associados à etnia, a um aumento nos índices de adiposidade de forma precoce, às síndromes genéticas, a um baixo peso no nascimento, desenvolvimento de doença endócrina, uso de medicações e baixo nível socioeconômico (POTIM JM, et al., 2017).

A obesidade pode estar associada a algumas síndromes genéticas, levando, na maioria das vezes, a criança a um quadro de desvios de fenotipos, apresentando baixa estatura, atraso cognitivo, puberdade tarde e atraso no desenvolvimento ósseo. As doenças endócrinas podem levar à obesidade muito mais por perda da estatura que realmente o ganho real de peso. No âmbito dessas doenças é importante lembrar do hipotireoidismo, deficiência de hormônio do crescimento (GH), síndrome de Cushing e da Síndrome dos Ovários Policísticos (SOP). Lesões hipotalâmicas como os próprios tumores e/ou tratamentos podem acarretar em um quadro de obesidade de difícil tratamento, normalmente associada à uma redução na velocidade de crescimento e em um atraso do desenvolvimento ósseo (PURNELL JQ, et al., 2018).

\section{Diagnóstico da obesidade infantil}

O diagnóstico de um paciente para risco de obesidade é relativamente simples. Os índices $\mathrm{P} / \mathrm{I}, \mathrm{E} / \mathrm{l}, \mathrm{IMC} / \mathrm{I}$ são obtidos de acordo com a idade e o sexo do indivíduo e a partir de dados antropométricos conferidos com rigor metodológico, como Peso $(P)$, Estatura $(E)$, circunferências corporais e "dobras. $O \mathrm{IMC} / \mathrm{I}$ é um parâmetro fácil de ser avaliado e é um forte preditor de risco metabólico em crianças. A circunferência abdominal também é um importante fator de risco para doença cardiovascular, dislipidemia e hipertensão arterial, além de ser indicativa de adiposidade abdominal ou central. O monitoramento desses índices pode ser uma estratégia potencial para identificar crianças e adolescentes com maior risco cardio-metabólico (RUIZ LD, et al., 2019).

O pediatra deve realizar um exame físico detalhado e uma boa anamnese para que se obtenha um diagnóstico precoce. Deve-se buscar identificar fatores que possivelmente estejam associados ao ganho de peso. Durante a anamnese é importante enfatizar a idade de início do ganho de peso, a presença de hipotonia nos primeiros meses de vida, as condições de nascimento, comorbidades prévias, uso de medicações (como medicações psicotrópicas e os glicocorticoides), alimentação, desenvolvimento puberal, prática de exercício físico e história familiar. No exame físico deverá ser avaliada a pressão arterial, o estádio puberal segundo Tanner, pesquisar os desvios fenotípicos e avaliar a presença de acantose nigricans. $\mathrm{Na}$ antropometria por sua vez, são coletados dados como estatura $(\mathrm{m})$, peso $(\mathrm{kg})$, cálculo do índice de massa corpórea (IMC, em $\mathrm{kg} / \mathrm{m}^{2}$ ), avaliação da distribuição da gordura (circunferência abdominal, em $\mathrm{cm}$ ) e velocidade de crescimento (em cm/ano) (SOCIEDADE BRASILEIRA DE PEDIATRIA, 2019).

De acordo com o Centro de Controle e Prevenção de Doenças (CDC), são considerados obesos adultos com IMC superior a $30 \mathrm{~kg} / \mathrm{m}^{2}$. Já adultos com IMC entre 25 e $30 \mathrm{~kg} / \mathrm{m}^{2}$ são classificados como sobrepeso. $\mathrm{Na}$ 
faixa etária pediátrica, isto e, entre 2 e 20 anos, são usadas para essas definições as curvas de percentil de IMC para idade e sexo. São considerados sobrepeso aqueles que se enquadram em percentil superior a 85 e são considerados obesos os com percentil superior a 95. Dentre os sinais antropométricos de alerta à ocorrência de obesidade estão o aumento do IMC em cerca de 3 a 4 unidades por ano ou mudança de canais percentuais/escore Z. Pode ser identificado risco de sobrepeso e obesidade mesmo quando o IMC ainda estiver compatível com a normalidade (CATALANO PM e SHANKAR, 2017).

\section{Manejo da obesidade infantil}

A obesidade pode ser dividida em dois tipos: a de origem exógena e a de origem endógena, sendo esta a mais frequente. No caso da endógena, a doença de base deve ser identificada e tratada. A obesidade exógena tem origem no desequilíbrio entre gasto calórico e ingestão, devendo ser manejada com orientação alimentar, especialmente otimização da prática de atividade física e mudanças de hábitos (PURNELL JQ, et al., 2018).

O tratamento deve ser introduzido de modo gradual e individual em conjunto com o paciente em questão e seus familiares. A instituição de planos alimentares muito restritivos não deve ser feita, com objetivo de aumentar a adesão do indivíduo ao plano terapêutico e evitar a perda de massa muscular e o retardo no crescimento. $O$ tratamento nutricional deve abranger uma dieta balanceada, com distribuição adequada de micro e macronutrientes e englobar uma orientação alimentar que permita a escolha de alimentos de ingestão habitual (RUIZ LD, et al., 2019).

É fundamental avaliar o tipo de alimento que é consumido na rotina desses pacientes, como e por quem eles são preparados e suas disponibilidades, compreender quais são os alimentos de preferência e as recusas, as atividades diárias da criança, o ambiente em que são realizadas as refeições, questionar se é realizada a ingestão de líquidos durante as refeições e em seus intervalos e questionar sobre tabus e crenças alimentares da família. Para a redução de peso diminuir tanto o consumo de alimentos e quanto a ingestão de dietas hipercalóricas já é suficiente. É muito importante que os familiares entendam que as crianças e os adolescentes seguem padrões paternos e, caso esses não sejam modificados ou manejados em conjunto, já é previsto um tratamento insatisfatório (PETER U, et al., 2018).

Em relação a dieta, é fundamental que ela determine a manutenção do peso ou sua perda controlada, quantidades adequadas para idade e sexo, crescimento e desenvolvimento normais, manutenção da massa muscular, hiporexia, se há traços de distúrbios psicológicos envolvidos e manutenção dos hábitos alimentares. As crianças menores devem perder menos peso para que o desenvolvimento não seja comprometido, para isso o ideal é que tenham pequeno ganho de peso ou que mantenham uma linearidade no mesmo (CATALANO PM e SHANKAR, 2017).

A supervisão da saúde é recomendada pelas diretrizes médicas identificando pacientes em risco através de peso ao nascer, história familiar, fatores socioeconômicos, comportamentais, culturais e étnicos. O IMC deve ser calculado e registrado uma vez ao ano em todas as crianças e adolescentes e as suas alterações devem ser utilizadas para identificar taxa excessiva de ganho de peso relativo ao crescimento linear. Além disso, o aleitamento materno deve ser encorajado; os pais e educadores devem ser orientados a promover padrões alimentares saudáveis, oferecendo lanches nutritivos; a autonomia das crianças no controle da sua ingestão alimentar deve ser encorajada, estabelecendo limites nas escolhas e deve ser oferecida rotineiramente a possibilidade de prática de atividade física. É de extrema importância a discussão de hábitos saudáveis por parte dos familiares, professores e profissionais da saúde com objetivo de auxiliar no controle da obesidade (RUIZ LD, et al., 2019).

\section{Complicações da obesidade infantil}

Dentre as diversas comorbidades associadas à obesidade existem fortes evidências de uma relação entre ela e um processo de inflamação, gerando um impacto negativo na resposta imunológica, processo aterosclerótico e doença cardiovascular. Na infância e na adolescência, o excesso de peso está associado diretamente com o perfil lipídico alterado e as concentrações elevadas de insulina plasmática, além de hipertensão arterial, esse conjunto de eventos pode levar ao surgimento prematuro de doença cardiovascular na vida adulta (FRUH SM, 2017). 
A obesidade infantil leva a inflamação vascular, predispondo a aterosclerose em uma idade precoce. $\mathrm{Na}$ infância são considerados alterados valores de pressão arterial sistólica e/ou diastólica acima do percentil 95 para sexo, idade e percentil de estatura e, identificam o estado de pré-hipertensão, valores entre o percentil 90 e 95. Outra comorbidade é o maior risco de lesões em membros inferiores, além disso, os pacientes obesos com fraturas do fêmur e da tíbia têm lesões mais graves, e predisposição a maior morbidade e mortalidade (SOCIEDADE DE PEDIATRIA DE SÃO PAULO, 2019).

O excesso de gordura em tronco e abdome, a quantidade de gordura total e aumento de gordura visceral são fatores importantes para o desenvolvimento de doenças crônicas. O risco de desenvolvimento de DM2 é triplicado com o sobrepeso. Assim como a obesidade, o hábito de fumar, o nível de colesterol aumentado e a presença HAS, DM2 ou sedentarismo são fatores de risco independentes para doença coronariana (STANFORD FC, et al., 2017).

A obesidade é fator de risco para dislipidemia, promovendo aumento de triglicerídeos e colesterol total, LDL e VLDL e redução da fração HDL. Com a perda de peso, melhora da alimentação e prática de exercícios físicos, o perfil lipídico global apresenta melhora, reduzindo dessa forma a chance de desenvolvimento de doenças cardiovasculares. A aterosclerose se inicia nos primeiros anos de vida, com o depósito de colesterol nas artérias, formando as estrias de gordura, as quais podem progredir para lesões ateroscleróticas avançadas em poucos anos (WANG Y, et al., 2017).

De modo geral, não é indicada a quantificação do perfil lipídico em crianças e adolescentes, mas em grupos de risco ela deverá ser realizada entre os 2 e 19 anos de idade. Uma vez confirmada a dislipidemia, a dieta deverá ser iniciada após os 2 anos e sempre priorizando as necessidades vitamínicas e energéticas da criança. Em situações que sejam necessários aumentar o percentual de gordura, isso deverá ser feito através da ingestão de alimentos ricos em gorduras monoinsaturadas (SOCIEDADE DE PEDIATRIA DE SÃO PAULO, 2019).

\section{Prevenção da obesidade infantil}

No pré-natal é recomendado que a gestante começe a realizar consultas pediátricas a partir de 32 semanas de gestação, o que dá início a formas de prevenção à obesidade infantil. Durante esse período é imprescindível que a mãe tenha uma boa orientação nutricional e um controle de perto sobre sua alimentação e seu ganho ponderal, o que torna fundamental o diálogo interdisciplinar para essa paciente. $O$ aleitamento materno deve ser incentivado a ser mantido de forma exclusiva até o sexto mês de vida e complementado até ao menos o segundo ano de vida. A amamentação é uma decisão que pode ser tomada estrategicamente antes da mulher engravidar, mas é durante o período gestacional que ela se torna mais adepta aos cuidados com a saúde, visando a proteção do bebê de complicações durante sua formação (TRANDAFIR LM e TEMNEANU OR, 2016).

A gestante deve ser instruída pelos profissionais da saúde sobre as diversas vantagens do aleitamento materno e seus benefícios para a saúde do bebê, da gestante e os que se estendem a toda uma sociedade, reduzindo consideravelmente a mortalidade infantil, levando a uma menor morbidade diminuindo as doenças crônicas e as doenças infecciosas causadas por inúmeros fatores protetores do leite humano. Pode reduzir a obesidade e suas comorbidades associadas, assim como melhor desempenho cognitivo (FLODMARK CE, 2018).

Idealmente, a amamentação deverá ser iniciada na primeira hora de vida, sempre que possível ainda na sala de parto. Sendo assim, o recém-nascido entra em contato precoce com as células maternas, sendo, em sua maioria, cerca de $80 \%$ delas, fatores imunomoduladores e macrófagos originados do sangue materno periférico, o que acaba facilitando o desenvolvimento do sistema imune da criança. No alojamento conjunto, é importante que a primeira mamada seja acompanhada por um profissional de saúde adequadamente treinado. No $7^{\circ}$ dia de vida é importante que a criança receba seu primeiro atendimento a nível ambulatorial, de forma precoce e por um pediatra, onde devem ser feitas orientações à mãe, a continuidade ao incentivo e o agendamento das consultas de retorno para que a criança seja acompanhada de perto (CATALANO PM e SHANKAR, 2017). 
As crianças de uma forma geral possuem maior risco para o desenvolvimento de obesidade nos primeiros quatro meses de vida. No sexto mês de vida recomenda-se o início da introdução alimentar complementar, fase em que é extremamente importante evitar suco de frutas, evitar adição de açúcar e derivados nos alimentos, dar preferência para as frutas in natura, evitar alimentos industrializados, avaliar a alimentação da família e incentivar o consumo de alimentos com baixo teor de gorduras, açúcar e sal. Leite de vaca ou de outros mamíferos não são recomendados durante os primeiros anos de vida quando não passaram por modificações, assim como os extratos vegetais (POTIM JM, et al., 2017).

O pediatra é peça fundamental para o atendimento ambulatorial das crianças e dos adolescentes de forma longitudinal de rotina, o que permite a intervenção diante de um quadro de obesidade em qualquer fase da sua vida. Em casos de obesidade infantil são necessárias o questionamento, a monitorização e o aconselhamento quanto a alguns aspectos sociais: medida de estatura, avaliação de peso corporal, medida de circunferência abdominal, avaliação do hábito alimentar, observação quanto ao estilo de vida do paciente e seus familiares, avaliação da pressão arterial, estímulo a prática regular de exercícios físicos, estabelecimento de rotinas para a criança ou o adolescente, estabelecer tempo máximo de exposição a tela, estabelecer horário de sono, avaliar e prescrever dieta habitual com qualidade e quantidade regradas, questionar e observar o ambiente das refeições e avaliar as atividades sociais e as situações de prazer (FLODMARK CE, 2018).

\section{CONSIDERAÇÕES FINAIS}

A obesidade infantil se configura atualmente como um grave problema de saúde pública em todo mundo. Existem diversos fatores que podem levar ao sobrepeso na criança, dentre eles estão os fatores genéticos, má alimentação e sedentarismo. Desta forma, são importantes o manejo e a prevenção da obesidade infantil, visto que pode ser facilmente controlada na grande maioria dos casos com mudança de hábitos alimentares e aumento da prática de exercícios físicos. Sabe-se que a obesidade está relacionada a diversas complicações e aumento da morbimortalidade infantil e também na faixa etária adulta, portanto, é de extrema importância a realização de um diagnóstico preciso e tratamento correto, bem como a instituição de políticas públicas para a prevenção da obesidade infantil.

\section{REFERÊNCIAS}

1. BARANOWSKI T, et al. Multi-etiological Perspective on Child Obesity Prevention. Current Nutrition Reports, 2019; 10.1007/S13668-019-0256-3.

2. CATALANO PM, SHANKAR K. Obesity and pregnancy: mechanisms of short term and long term adverse consequences for mother and child. The BMJ, 2017;356:j1.

3. CESARE MD, et al. The epidemiological burden of obesity in childhood: a worldwide epidemic requiring urgent action. BMC Medicine, 2019; 17(1):212.

1. FLODMARK CE. Prevention Models of Childhood Obesity in Sweden, Obesity Facts, 2018;11(3):257-262.

2. FRUH SM. Obesity: Risk factors, complications, and strategies for sustainable long-term weight management. Journal of the American Association of Nurse Practitioners, 2017; 29(S1):S3-S14.

3. NITTAR G, et al. Fighting obesity in children from European World Health Organization member states. Epidemiological data, medical-social aspects, and prevention programs. Clinical Therapeutics, 2019; 170(3):e223e230.

4. PETER U, et al. Obesity and asthma. The Journal of Allergy and Clinical Immunology, 2018; 141(4):1169-1179.

5. POTIM JM, et al. Ultra-processed Food Intake and Obesity: What Really Matters for Health-Processing or Nutrient Content? Current Obesity Reports, 2017; 6(4):420-431.

6. PURNELL JQ, et al. Definitions, Classification, and Epidemiology of Obesity. Endotext, 2018; 2(1):1-5.

7. RUIZ LD, et al. Adolescent Obesity: Diet Quality, Psychosocial Health, and Cardiometabolic Risk Factors. Nutrients, 2019; 12(1):43.

8. SOCIEDADE BRASILEIRA DE PEDIATRIA. Orientação manual de obesidade na infância e adolescência, Departamento de Nutrologia, 2019; 3ㄹed. Departamento de Nutrologia, 2019. Disponível em: https://www.sbp.com.br/fileadmin/user_upload/Manual_de_Obesidade_-_3a_Ed_web_compressed.pdf. Acesso em 22 set 2021. 
9. SOCIEDADE DE PEDIATRIA DE SÃO PAULO. Enfrentando a obesidade infantil. Pediatria actualize-se, $2019 ; 4(2): 5$. Disponível em: https://www.spsp.org.br/site/asp/boletins/AtualizeA4N2.pdf. Acesso em 22 set 2021.

10. STANFORD FC, et al. Media and Its Influence on Obesity.Current Obesity Reports, 2018; 7(2):186-192.

11. STYNE DM. Pediatric Obesity-Assessment, Treatment, and Prevention: An Endocrine Society Clinical Practice Guideline. The Journal of Clinical Endocrinology and Metabolism, 2017; 102(3):709-757.

12. TRANDAFIR LM, TEMNEANU OR. Pre and post-natal risk and determination of factors for child obesity. Journal of Medicine and Life, 2016; 9(4):386-391.

13. WANG Y, et al. A Systematic Examination of the Association between Parental and Child Obesity across Countries. Advances in Nutrition, 2017;8(3):436-448. 